\title{
Penetration of cranial nerves by intracranial arteries and veins: a comprehensive review
}

\author{
Galyna Ivashchuk ${ }^{1}$, Andrea Andrea ${ }^{2}$, Erfanul Saker ${ }^{1}$, Marios Loukas $^{1}$, Rod J. Oskouian $^{3}$, R. Shane Tubbs ${ }^{2}$ \\ ${ }^{\prime}$ Department of Anatomical Sciences, St. George's University, Grenada, West Indies \\ ${ }^{2}$ Seattle Science Foundation, Seattle, WA, USA \\ ${ }^{3}$ Swedish Neuroscience Institute, Swedish Medical Center, Seattle, WA, USA
}

\begin{abstract}
The relationship between cranial nerves and surrounding blood vessels is important to surgeons who operate in this region and radiologists who interpret cranial imaging. However, only sparse information is available regarding the potential for intracranial vessels to pierce cranial nerves. Therefore, the current comprehensive review was performed. Some reports do exist that describe this variant anatomy. Both veins and arteries have been reported to rarely pierce cranial nerves. Clinicians and surgeons should be aware of this possible derailment of normal anatomy as it could affect their patients or result in symptoms that do not have a precise etiology.
\end{abstract}

Keywords: cerebral arteries; cerebral veins; cranial nerves

Anatomy 2016;10(2):143-147 @2016 Turkish Society of Anatomy and Clinical Anatomy (TSACA)

\section{Introduction}

The close anatomical relationship between intracranial nerves and cerebral vessels has long been discerned. While the intracranial nerves and cerebral vessels are normally separated entities, the two structures can be intertwined on some rare occasions. This can lead to the penetration of the nerve by the vessels, followed subsequently by complications such as aneurysms. A few theories have been postulated as to why this phenomenon happens. Beatty ${ }^{[1]}$ proposed three mechanisms by which this can evolve: 1) aneurysm enlarges along congenital splitting of the nerve, 2) aneurysm originates from a vessel already penetrating a nerve, 3) aneurysm directly penetrates the nerve. Alternatively, Towbin and Kanal ${ }^{[2]}$ suggested an explanation for why arterial fenestrations are more common than venous speculating arterial aneurysm formations at fenestrations are largely due to turbulent flow at bifurcation sites, and defects in the media at both proximal and distal branch points.

Recently, new nomenclature has been used to differentiate between a duplicated and a fenestrated vessel. ${ }^{[3]} \mathrm{A}$ duplication of a cerebral vessel occurs when branches of anomalous vessel remain separate over the entire course, while fenestrated vessels are those in which the branched vessels reunited into a single normal vessel. In these fenestrated vessels, cranial nerves pass through in the space between the aberrant branches.

A profound understanding regarding the anatomy of the cerebral vessels and intracranial nerves is rendered critical to ensure both the safety and efficiency of clinical procedures as well as to better understand the anatomical structure. This paper serves as a comprehensive review of what has been known about the relationship between cranial nerves and the cerebral vessels.

\section{Arteries Piercing Cranial Nerves}

\section{Optic nerve}

Few arteries have been reported in the literature to penetrate the optic nerve. Herein we discuss the internal carotid-ophthalmic artery and anterior communicating artery, two arteries that have been implicated in the penetration of cranial nerves.

Internal carotid-ophthalmic artery

Internal carotid artery (ICA)-ophthalmic artery aneurysms constitute $0.3 \%$ to $1 \%$ of intracranial aneurysms. ${ }^{[4]}$ The 
directions towards which such aneurysms are directed mostly depend on the size of the arteries; small arteries usually project upwards. Carotid-ophthalmic artery aneurysms mostly arise from medial or superior medial wall of the internal carotid artery between the ophthalmic and posterior communicating arteries; ${ }^{[5]}$ however, in their study Kanamaru et al. ${ }^{[6]}$ reported a carotid-ophthalmic artery aneurysm case that arose from the anterior (instead of the medial or superior) wall. As the aneurysms enlarge, they extend medially likely due to the optic nerve restraint. ${ }^{[1]}$

It is rare, however, for these aneurysms to penetrate or physically bifurcate the optic apparatus. ${ }^{[4]}$ Only a limited number of case reports illustrating optic nerve penetration by an ophthalmic artery aneurysm have been reported in literature. ${ }^{[1,5,7]}$ An outstanding case of carotid-ophthalmic artery aneurysm was reported by Beatty ${ }^{[1]}$ in 1986, in which a giant carotid-ophthalmic artery was pointing directly upwards, splitting the optic nerve open over the length of 5-7 mm. More recently, Takagi and Miyamoto ${ }^{[4]}$ reported a case of an internal carotid-ophthalmic artery aneurysm piercing the optic nerve along with the falciform ligament, the first documented case of such an occurrence.

\section{Anterior communicating artery aneurysms}

Anterior communicating artery (AComA) aneurysms have also been reported to cause the piercing of the optic nerve. ${ }^{[8]}$ In 1997, Date et al..$^{[9]}$ published a study that involved a $7 \mathrm{~mm}$ inferior projection of AComA aneurysm, in which the thrombosed dome penetrated the right half of the optic chiasm and compressed the right optic tract. Treatment was attempted by aneurysmal clipping; however visual defect persisted post-procedure. ${ }^{[9]}$ Similarly, Lee et al. ${ }^{[10]}$ described the penetration of the optic chiasm and right optic nerve by a recurrent AComA aneurysm, where previous treatments were attempted by means of detachable coils.

AComA aneurysm can also originate from within the optic nerve, in which the artery splits the optic nerve into medial and lateral portions. ${ }^{[11]}$

Post mortem brain analyses have revealed saccular aneurysms extending into the optic nerve, either from cerebral or internal carotid artery and compressing the nerve severely, turning it into a C-shaped structured, which was completely covered by aneurysm. ${ }^{[12]}$

\section{Symptoms}

Aneurysmal rupture presents with typical symptoms of subarachnoid hemorrhage, including headache, dizziness, neck pain, and nonspecific symptoms. Some patients also experience dysphasia, clumsiness of the upper extremities, and in extreme cases are found comatose. ${ }^{[1,7]}$ Visual disturbances are commonly reported, however, it is not always present in some cases. ${ }^{[1,1]}$ The presence of visual symptoms suggests either a sudden disruption of nerve integrity from the abrupt aneurysm rupture through an intact nerve or a sudden enlargement of any aneurysm through a de novo slit. ${ }^{[7]}$

Anterior communicating artery aneurysms commonly cause subarachnoid hemorrhage, but rarely visual disturbances. Date et al. reported a case of abrupt onset blurred vision in both eyes, in addition to headache, left homonymous hemianopsia and right visual acuity disturbance. ${ }^{[9]}$

Only $18 \%$ of patients with internal carotid-ophthalmic artery aneurysms experience visual disturbances; some experience lower quadrantonopsia and decrease visual acuity. ${ }^{[4,6]}$ When visual symptoms are experienced, they do not develop until later in the onset of the condition. Visual loss occurs in $1 / 3$ of all reported aneurysm cases and is considered related to the direct compression or injury at the time of rupture. ${ }^{[13]}$ In some instances, part of optic nerve has to be cut in order to properly clip the aneurysm, with resultant visual defects. ${ }^{[1]}$

\section{Origin}

Date et al. ${ }^{[9]}$ proposed that the penetration of the optic nerve progresses from subarachnoid hemorrhage. This phenomenon causes a small stream of blood under arterial pressure to excavate through CNIII, create a gap between fibers of the nerve, therefore allowing the aneurysm to herniate from the new orifice through the nerve. ${ }^{[9]}$ Alternatively, Kanamaru et al. ${ }^{[6]}$ proposed that aneurysm develops fairly early in development, on the fetal vessels that supply the embryonic optic apparatus; the aneurysm later progresses to penetrate the optic nerve or chiasm. Lastly, Fujita et al. ${ }^{[5]}$ suggested that the complete penetration of optic chiasm is due to the rapid growth of an unruptured aneurysm, leading to rapidly-progressing visual symptoms.

\section{Oculomotor nerve}

Just as the case with the optic nerve, aneurysms have also been identified as the common culprits of the penetration of the oculomotor nerve by arteries.

The artery splits an oculomotor nerve into proximal (from cerebral peduncles to just distal to anastomosis of posterior cerebral and posterior communicating artery), middle (to entrance of cavernous sinus) and distal (within the cavernous sinus) sections. ${ }^{[14]}$ The nerve's proximal segment is commonly penetrated by the circumflex, thalamoperforating, or both arteries, however, no such penetration was reported in the middle and distal segments. ${ }^{[14]}$

The penetration of oculomotor nerves by circumflex mesencephalic arteries or the branch(es) of perforating 
vessels of the posterior cerebral artery accounted for up to $60 \%$ of all the brains examined. ${ }^{[15]}$

Long or short circumflex mesencephalic arteries account for $69 \%$ of vessels that penetrate CNIII. These arteries originate from medial or dorsal surface of the initial segment of the posterior cerebral artery; their sizes range between $0.3-0.7 \mathrm{~mm}$. Branches of the interpeduncular perforating posterior cerebral arteries account for $31 \%$ of vessels; their diameter are considerably thinner $(0.1-0.5 \mathrm{~mm})$. Both types of arteries branch off further into tiny arterioles that go into the oculomotor nerve. In most cases, only one vessel pierces the nerve; however, Milisavljevic et al. reported that a nerve was pierced by two branches of the long circumflex mesencephalic artery. ${ }^{[15]}$ In addition, both arteries are known to always run through the proximal part of the CNIII, either through nerve itself or between its root fibers. Penetrations most commonly occur at an acute angle.

The aberrant duplicate posterior cerebral artery (PCA) branch entered and traversed the split oculomotor nerve at a region where aneurysm arose. Binning and Couldwell ${ }^{[16]}$ reported a case of an aberrantly duplicated PCA aneurysm, in which the dome that was embedded within the nerve's split fibers, projected inferiorly into the oculomotor nerve.

Atherosclerotic stenosis or occlusion has been identified as the rare causes of de novo aneurysm. ${ }^{[17]}$ They induce degenerative breakdown of the vascular wall and promote formation of fibrous atheroma and luminal stenosis, leading to weaker blood flow and turbulent effects, and finally resulting in a wide variation of wall shear stresses. ${ }^{[17]}$ In 1997, Horiuchi et al. ${ }^{[18]}$ reported a de novo aneurysm of the right internal carotid-posterior communicating artery within the fenestrated oculomotor nerve. Shin et al. ${ }^{[17]}$ reported de novo posterior communicating artery aneurysm in a patient with severe atherosclerotic stenosis of the ICA; the patient's arterial dome projected posterolaterolaterally into the penetrating oculomotor nerve.

With the exception of a pre-operative MRI diagnosis reported by Jea et al. ${ }^{[19]}$ in 2003 , all cases of oculomotor nerve penetration by arterial aneurysm were discovered intra-operatively or post mortem.

\section{Symptoms}

Oculomotor paresis is one of the hallmark symptoms of IC-PCA aneurysm, commonly caused by local compression of the oculomotor nerve growth of aneurysm or by hemorrhagic dissection of the nerve. ${ }^{[18]}$ In some cases, no oculomotor symptoms were experienced prior to surgery ${ }^{[16]}$ while in others, the following symptoms prevailed: headache, sudden onset of left oculomotor paresis, diplopia, lid ptosis, dysfunction of adduction and corresponding pupil dilation ${ }^{[18,20]}$ Binning and Couldwell reported that no intraoperative damage was done to the nerve ${ }^{[16]}$ however, Horiuchi et al. ${ }^{[18]}$ was forced to sacrifice part of the fenestrated oculomotor nerve.

\section{Trochlear nerve}

The trochlear nerve has been reported to pass through an arterial ring consisting of the rostral and caudal trunk of superior cerebellar artery. ${ }^{[21]}$ However, due to its small size, only arteries that are less than $0.2 \mathrm{~mm}$ thick can course through these nerve filaments. ${ }^{[2]}$

\section{Facial nerve}

A case study reported a $2 \mathrm{~mm}$ penetration of the distal portion of the facial nerve root exit zone by the anterior inferior cerebellar artery (AICA). ${ }^{[23]}$ The patient experienced atypical progressive symptoms of hemifacial spasm that progressed from the mouth to the eye, and finally the platysma after 4 years. Symptoms were reported to have been triggered by eating or drinking, however patient had no cranial nerve palsy or neurological deficits. Liu and Fagan ${ }^{[24]}$ reported an unanticipated intraoperative finding where the AICA traveled through the facial nerve near the brainstem, yet it was somehow symptom-free.

\section{Glossopharyngeal nerve}

In 2015, Ikeda et al. ${ }^{[25]}$ reported two cases of glossopharyngeal neuralgia caused by a perforating posterior inferior cerebellar artery that compressed the glossopharyngeal rootlet.

\section{Hypoglossal nerve}

The fenestration and segment duplication of basilar arteries are very rare. These phenomena involve the substitution of an arterial segment by two compartments separated by a window. In their study, de Caro et al. ${ }^{[2]}$ performed a post mortem examination on a 68 -year-old male who died from a myocardial infarction was examined post mortem, in which the luminal septum of his right vertebral artery was made of tightly positioned arterial walls with a small bundle of nerve fibers passing through it. De Caro et al. suggested two mechanisms by which vertebral artery can be duplicated: with or without fenestration. ${ }^{[2]}$ In the duplication with fenestration, a segment of the vertebral artery is substituted by two branches separated by a window persisting from the caudal part of the primitive lateral vertebrobasilar anastomosis. The latter involves the presence of luminal septum, and is thought to be caused by the incomplete fusion of vessels of the primitive occipital plexus. ${ }^{[2]}$ The anomaly of the caudal rootlet is also likely to predispose the development of atherosclerotic lesions and the occurrence of vascular thrombosis. ${ }^{[26]}$ 


\section{Veins piercing cranial nerves}

\section{Abducens nerve}

In $15 \%$ of examined brains, the abducens nerve is penetrated by the corresponding pontine veins (anterolateral pontine or tributary of the anteromedian pontine vein). ${ }^{[15]}$ In all cases, the vein always courses through the middle of the initial part of the nerve; its mean vessel diameter was reported to be $1 \mathrm{~mm} \cdot{ }^{[15]}$

\section{Facial nerve}

Cvetko $^{[27]}$ reported a case in which the cervical branch of the facial nerve passed through a large $(6 \mathrm{~cm})$ fenestration of the external jugular vein. It was speculated that this was due to a derangement in early embryonic development (between 3rd - 6th weeks of gestation) and that of vessel duplication, during which the nerve was trapped in the fenestrated vein. ${ }^{[27]}$

\section{Accessory nerve}

The understanding of the accessory nerve anatomy and its variations is especially important for the recent advances in surgical neck dissections to treat metastatic head and neck cancers. ${ }^{[28]}$ Among others, the internal jugular vein (IJV) and the accessory nerve are considered the most important structures in neck dissections, as they provide notable intraoperative landmarks, hence enhancing surgical precision and accuracy. ${ }^{[2]}$ Accessory nerve injuries can lead to severe post-operative morbidities, including chronic shoulder pain and weakness. ${ }^{[2]]}$ The passing of the accessory nerve through a fenestrated internal jugular vein was reported in $0.9 \%-2.8 \%$ of reported cases. ${ }^{[10,28,30]}$ In all cases, the nerve always passed medially to the anterior part and laterally to the posterior part of the fenestrated IJV. Scholars proposed that the IJV duplication is likely to originate from an inadequate condensation of the embryonic capillary plexus, a structure developing posterior to the precardial veins. ${ }^{[28]}$ Most of the abnormalities within IJV are unilateral. ${ }^{[3]}$

There have been seven cases of the accessory nerve passing through the fenestration of internal jugular vein that have been reported. ${ }^{[2,31,32]}$

\section{Conclusion}

Cranial nerve penetration by cerebral vessels is a rare phenomenon, but important to recognize intraoperatively and on imaging, when seen. Such knowledge will allow surgeons to avoid long-term complications and accidental injury to these structures.

\section{References}

1. Beatty RA. Splitting of the optic nerve by a carotid-ophthalmic artery aneurysm. J Neurosurg 1986;65:560-2.

2. Towbin AJ, Kanal E. A review of two cases of fenestrated internal jugular veins as seen by CT angiography. AJNR Am J Neuroradiol 2004;25:1433-4.

3. Downie SA, Schalop L, Mazurek JN, Savitch G, Lelonek GJ, Olson TR. Bilateral duplicated internal jugular veins: case study and literature review. Clin Anat 2007;20:260-6.

4. Takagi Y, Miyamoto S. Penetration of the optic nerve and falciform ligament by an internal carotid artery-ophthalmic artery aneurysm: case report. Neurol Med Chir (Tokyo) 2014;54:211-3.

5. Fujita A, Tamaki N, Yasuo K, Nagashima T, Ehara K. Complete penetration of the optic chiasm by an unruptured aneurysm of the ophthalmic segment: case report. Surg Neurol 2002;57:130-4.

6. Kanamaru K, Ishida F, Taki W. Splitting and penetration of the optic nerve by an aneurysm arising from the anterior wall of internal carotid artery: case report. J Neurol Neurosurg Psychiatry 2001;71: $525-7$.

7. Wang YY, Thani NB, Han TF. Optic nerve penetration by a carotico-ophthalmic artery aneurysm. J Clin Neurosci 2010;17:931-3.

8. Mäurer J, Mäurer E, Perneczky A. Surgically verified variations in the A1 segment of the anterior cerebral artery. Report of two cases. J Neurosurg 1991;75:950-3.

9. Date I, Akioka T, Ohmoto T. Penetration of the optic chiasm by a ruptured anterior communicating artery aneurysm. Case report. J Neurosurg 1997;87:324-6.

10. Lee SH, Koh JS, Lee CY, Kwon GY, Ryu CW. Penetration and splitting of the optic apparatus by intrasaccular coils within an anterior communicating artery aneurysm. Clin Neurol Neurosurg 2011;113:578-81.

11. Joo SP, Kim TS, Seo BR. Splitting of the oculomotor nerve by the posterior communicating artery-case report. Neurol Med Chir (Tokyo) 2010;50:57-8.

12. Milliser RV, Greenberg SR, Neiman BH. Congenital or berry aneurysm in the optic nerve. J Clin Pathol 1968;21:335-8.

13. Norwood EG, Kline LB, Chandra-Sekar B, Harsh GR 3rd. Aneurysmal compression of the anterior visual pathways. Neurology 1986;36:1035-41.

14. Cahill M, Bannigan J, Eustace P. Anatomy of the extraneural blood supply to the intracranial oculomotor nerve. Br J Ophthalmol 1996;80:177-81.

15. Milisavljeviç M, Marinkoviç S, Loliç-Draganiç V, Kovaceviç M. Oculomotor, trochlear, and abducens nerves penetrated by cerebral vessels. Microanatomy and possible clinical significance. Arch Neurol 1986;43:58-61.

16. Binning MJ, Couldwell WT. Fenestration of the oculomotor nerve by a duplicated posterior cerebral artery and aneurysm. Case report. J Neurosurg 2009;111:84-6.

17. Shin HS, Lee SH, Koh JS. Asymptomatic penetration of the oculomotor nerve by a de novo aneurysm aneurysm associated with severe atherosclerotic stenosis of the supraclinoid internal carotid artery. J Korean Neurosurg Soc 2014;56:48-50.

18. Horiuchi T, Kyoshima K, Oya F, Kobayashi S. Fenestrated oculomotor nerve caused by internal carotid-posterior communicating artery aneurysm: case report. Neurosurgery 1997;40:397-9.

19. Jea A, Başkaya MK, Morcos JJ. Penetration of the optic nerve by an internal carotid artery-ophthalmic artery aneurysm: case report and literature review. Neurosurgery 2003;53:996-1000. 
20. Toyota S, Taki T, Wakayama A, Yoshimine T. Unruptured internal carotid-posterior communicating artery aneurysm splitting the oculomotor nerve: a case report and literature review. J Neurol Surg Rep 2014;75:e180-2.

21. Rusu MC, Vrapciu AD, Patraşcu JM. Variable relations of the trochlear nerve with the pontomesencephalic segment of the superior cerebellar artery. Surg Radiol Anat 2015;37:555-9.

22. Lang J, Reiter W. Medical findings on the trochlear nerve. [Article in German] J Hirnforsch 1986;27:101-10.

23. Oh CH, Shim YS, Park H, Kim EY. A case of hemifacial spasm caused by an artery passing through the facial nerve. J Korean Neurosurg Soc 2015;57:221-4.

24. Liu R, Fagan P. Unusual course of the anterior inferior cerebellar artery through the facial nerve in the cerebellopontine angle. Otolaryngol Head Neck Surg 2001;124:479.

25. Ikeda N, Toda H, Yamamoto M, Kanemaru S, Ishikawa M, Iwasaki $\mathrm{K}$. A perforating artery compressing the nerve rootlet and causing glossopharyngeal neuralgia. Neurosurgery 2015;11:382-6.

26. de Caro R, Parenti A, Munari PF. Course of the caudal hypoglossal rootlet through the vertebral artery. J Anat 1995;187:499-501.
27. Cvetko E. A case of unilateral fenestration of the external jugular vein, through which the cervical branch of the facial nerve passes. Anat Sci Int 2013;88:151-2.

28. Hashimoto Y, Otsuki N, Morimoto K, Saito M, Nibu K. Four cases of spinal accessory nerve passing through the fenestrated internal jugular vein. Surg Radiol Anat 2012;34:373-5.

29. Gardiner KJ, Irvine BW, Murray A. Anomalous relationship of the spinal accessory nerve to the internal jugular vein. Clin Anat 2002;15: $62-3$.

30. Taylor CB, Boone JL, Schmalbach CE, Miller FR. Intraoperative relationship of the spinal accessory nerve to the internal jugular vein: variation from cadaver studies. Am J Otolaryngol 2013;34:527-9.

31. Oztürk NC, Talas DÜ. Fenestration of internal jugular vein and relation to spinal accessory nerve: case report and review of the literature. Clin Anat 2010;23:883-4.

32. Prades JM, Timoshenko A, Dumollard JM, Durand M, Merzougui N, Martin C. High duplication of the internal jugular vein: clinical incidence in the adult and surgical consequences, a report of three clinical cases. Surg Radiol Anat 2002;24:129-32.

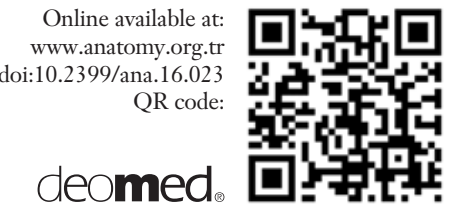

Correspondence to: Galyna Ivashchuk, MD Department of Anatomical Sciences,

St. George's University, Grenada, West Indies

Phone: +12067326510

e-mail: givashchuk@sgu.edu

Conflict of interest statement: No conflicts declared.

This is an open access article distributed under the terms of the Creative Commons Attribution-NonCommercial-NoDerivs 3.0 Unported (CC BY-NCND3.0) Licence (http://creativecommons.org/licenses/by-nc-nd/3.0/) which permits unrestricted noncommercial use, distribution, and reproduction in any medium, provided the original work is properly cited. Please cite this article as: Ivashchuk G, Andrea A, Saker E, Loukas M, Oskouian RJ, Tubbs RS. Penetration of cranial nerves by intracranial arteries and veins: a comprehensive review. Anatomy 2016;10(2):143-147. 